\title{
HILFE FÜR ÄLTERE MENSCHEN IM RAHMEN DER SOZIALARBEIT IN POLEN (POMOC DLA OSÓB STARSZYCH W RAMACH PRACY SOCJALNEJ W POLSCE)
}

\begin{abstract}
Streszczenie
We współczesnym zróżnicowanym społeczeństwie coraz częściej występuje brak porozumienia interpersonalnego, a człowiek żyje w ciagłych zmianach i pośpiechu. Trzeba przyznać, iż w społeczeństwie szybkiego rozwoju i przemian żyje się trudno, zwłaszcza osobom starszym, które czesto wchodzac w tzw. okres starzenia czuja się odrzucone, niepotrzebne.

Jedna z form pomocy człowiekowi, również w okresie starzenia sie jest praca socjalna. Dlatego też niniejszy artykut statuuje się w obszarze wsparcia seniorów i dotyczy wyjaśnienia podstawowych zagadnień dotyczacych: form pomocy wobec osób starszych oraz roli pracownika socjalnego $w$ zakresie wsparcia seniorów. Celem jest próba ukazania wartości świadczeń pracy socjalnej oferowanych osobom starszym (seniorom) jako instrumentów pomocy $i$ wsparcia tej grupy osób.
\end{abstract}

Słowa kluczowe: starość, seniorzy, praca socjalna, wsparcie, pomoc, świadczenia

\section{Einführung}

Die heutige Gesellschaft ist ein sehr differenzierter Organismus. Immer öfter kommt es zu Mängeln an zwischenmenschlicher Verständigung, und der Mensch lebt unter Bedingungen ständiger Veränderungen und der Eile. Eine fürwahr für

* Józef Młyński - dr n. hum. w zakresie socjologii, adiunkt na Wydziale Studiów nad Rodziną, Uniwersytet Kardynała Stefana Wyszyńskiego w Warszawie. 
die Entwicklung diesen Organismus wichtige Gruppe sind Senioren, welche aus dem Grund des Problems der Alterung eine Herausforderung für die heutigen Gesellschaften darstellen. Die wichtigsten Phänomene und gesellschaftliche Prozesse, welche die Funktionsweise der immer älter werdenden Gesellschaften beeinflussen, sind: die sich stetig verändernde durchschnittliche Lebensdauer, „welche zur Verlängerung der Phase des hohen Alters beiträgt, wenn die Schwelle des demografischen, ökonomischen Übergangs in die Zeit des Alters sich nicht verändert, die womöglich die Notwendigkeit des Umdenkens der Altersschwelle mit sich bringt (z. B. Pensionsalter, demografischer Schwellen) und ihre Verschiebung weiter nach Oben auf der Zeitskala erfordert; ferner die Erhöhung der absoluten Zahlen der älteren Menschen (wobei sich nicht nur ihr Anteil an der Gesellschaft, aber auch ihre Zahl, erhöht); die Änderungen der Proportionen zwischen der Altersgruppen, wobei der Anteil älterer Menschen an der allgemeine Bevölkerungszahl wächst" ${ }^{\text {"1 }}$.

In einer von schneller Entwicklung und schnellen Veränderungen geprägten Gesellschaft ist es schwierig zu leben, umso wahrer ist es für ältere Personen, welche oft bei Antritt der sog. Alterungsperiode sich verworfen und überflüssig fühlen. Eine der Formen der Hilfe für Menschen ist Sozialarbeit, deren Wirkung die Wiederbringung der ordentlichen Funktionsweise in der Gesellschaft ist. Dieser Beitrag positioniert sich in dem Bereich der Unterstützung älterer Menschen im Bereich der Hilfe für Senioren, und befasst sich mit der Klärung grundlegender Fragen, Hilfsformen der Sozialarbeit gegenüber ältere Menschen sowie der Rolle des Sozialarbeiters im Bezug auf Seniorenunterstützung. Das Ziel dabei ist ein Versuch der Versinnbildlichung des Wertes der Sozialhilfeleistungen, welche älteren Personen (Senioren) als Hilfswerkzeuge angeboten werden sowie der Unterstützung dieser Personengruppe im Allgemeinen.

\section{Begriffsklärung: Gerontologie, Alter, Sozialarbeit}

Mitte des 20. Jahrhunderts kam eine neue Wissenschaft zur Welt: Die Gerontologie. Sie befasst sich mit den Erscheinungen und Problemen im Bezug auf das Alter, das selbst eine heterogene Periode darstellt. Alter beginnt und dauert bei jeder Person anders. Er hängt wesentlich von der beruflichen Aktivität, Lebensbedingungen, vom Sozialstatus und physischer Aktivität ab. Dieser Zeitraum lässt sich in drei Abschnitte Unterteilen: Dabei unterteilt wird die Gruppe in ältere Menschen, in einem sozusagen frühen hohen Alter, zwischen dem 60. und 70.

1 B. Szatur-Jaworska, P. Błędowski, M. Dzięgielewska, Podstawy gerontologii społecznej, Warszawa 2006, s. 242. 
Lebensjahr; die zweite Gruppe sind alte Menschen - zwischen dem 75. und 90. Lebensjahr, wonach folgen sehr alte Menschen, welche Zeit auch als Langlebigkeit bezeichnet werden kann - nach dem 90. Lebensjahr ${ }^{2}$. Zusammen mit der Alterung, welche Zeit eine schöne, wertvolle sein sollte, erscheinen auch gesellschaftliche Probleme, wie z. B. die schlechte ökonomische Lage der Senioren, begrenzter Zugang zu Gesundheitsvorsorge, unzulängliche Vorbereitung der Personen auf das Alter und fehlende Vorbereitung der Gesellschaft auf das Phänomen des Alters.

Das Alter ist ein sehr weit gefasster Begriff. Nach A. Zych ist es eine unvermeidliche Existenzperiode, in welcher die biologischen, psychischen sowie sozialen Prozesse synergisch wirken, indem sie zur Beeinträchtigung des mit der Reduktion der Lebensfunktionen und einer Reihe morphologischer Änderung verbundenen biologischen und psychischen Gleichgewichts führen ${ }^{3}$. P. Błędowski wiederum interpretiert diesen Zeitraum als eine „Phase des Menschenlebens, in welcher negative Veränderungen, die sich mit der biologischen Schwächung des Körpers und der Reduktion der psychologisch-physischen Leistungsfähigkeit verbinden, von Veränderungen des sozialen Status, der ökonomischen Situation, der beruflichen Aktivität und der Familienlage, begleitet werden ${ }^{64}$.

Die oben angeführten Definitionen unterstreichen die Tatsache, dass das Alter ein unvermeidlicher Prozess ist, wobei viele Veränderungen nicht nur am menschlichen Körper, aber auch an der Stellung des Menschen in der Gesellschaft, am Status und an den erfüllten gesellschaftlichen Rollen auftreten. Die wichtigsten Unbequemlichkeiten für Senioren sind: Das Gefühl des Überflüssigseins, Verwerfung, Einsamkeit, Behinderung, Krankheiten. Diese Probleme verursachen, dass ein älterer Mensch sich unnötig und nutzlos fühlt, daher tritt er oder sie von den Angelegenheiten der Gesellschaft zurück und sich von anderen isoliert. Daher brauchen Menschen im fortgeschrittenen Alter besondere Sorge, Kummer, welche von Sozialarbeit geleistet werden können.

Sozialarbeit wird verschiedenartig aufgefasst. Im Polnischen Sozialhilfegesetz (Pl. Ustawa o Pomocy Społecznej) wird sie folgendermaßen interpretiert: Es ist „berufliche Aktivität, welche auf die Hilfe für Personen oder Familien gerichtet ist, die Möglichkeit ihres Funktionierens in der Gesellschaft zu stärken oder wiederherzustellen, sowie der Herstellung der Bedingungen, welche den Weg zu

\footnotetext{
Vgl. J. Kędzior, Współczesne wyzwania pracy socjalnej, Toruń 2006, s. 169.

3 Vgl. A. Zych, Słownik gerontologii społecznej, Warszawa 2001, s. 202. Vgl. E. Rosset, Ludzie starzy. Studium demograficzne, Warszawa 1967, s. 12.

4 P. Błędowski, Lokalna polityka społeczna wobec ludzi starszych, Warszawa 2002, S. 63.
} 
diesem Ziel begünstigen“" . Nach der Generalversammlung der Internationalen Föderation der Sozialarbeiter ist „Sozialarbeit ein Beruf, der soziale Veränderungen sowie Lösung der bei zwischenmenschlichen Beziehungen entstehenden Probleme, sowie die Stärkung und Befreiung von Menschen zum Zwecke der Bereicherung ihres Wohlseins unterstützt" ${ }^{\text {“6 }}$.

Der Ausschuss des Europarats gibt an, dass „Sozialarbeit eine besondere berufliche Tätigkeit ist, welche sich als Ziel die bessere gegenseitige Adaptation von Personen, Familien, Gruppen sowie der sozialen Umgebung, in welcher diese leben, sowie die Entwicklung der persönlichen Würde und Verantwortung der Einzelpersonen auf dem Wege der Berufung auf potenzielle Möglichkeiten der einzelnen Personen zu inter-beruflichen Beziehungen, aber auch zu sozialen Kräften und Mitteln, setzt" ${ }^{\text {"7 }}$.

Aus den oben erwähnten Definitionen geht hervor, dass das Ziel der Sozialarbeit die Leistung von Unterstützung und Hilfe ist: Für Einzelpersonen, Familien, Gruppen und Lokalgesellschaften, zum Zweck der Rückkehr ins richtige Funktionieren in der Gesellschaft. In das Konzept der durch Sozialarbeit angebotenen Hilfe passt die Gruppe der Senioren hinein. „Diese Gruppe stellt eine besondere Herausforderung für Sozialarbeiter dar. Arbeit mit Senioren erfordert von den Sozialarbeitern besonderes Wissen über Sozialumstände, welche oft Druck auf ältere Menschen ausüben, sowie das Wissen über den Prozess des Alterns sowie über die Veränderungen, welche im Zuge dieses Prozesses vorkommen. Ein Sozialarbeiter soll den Senioren und ihren Familien Angaben zu den verfügbaren Leistungen, aber auch zu den Unterstützungsmöglichkeiten liefern“" .

\section{Sozialarbeit und Unterstützung ältere Menschen}

Hilfe und Unterstützung sind permanente Hilfeformen für diejenigen, welche in Extremsituationen und schwierige Situationen geraten sind. Nach A. Chabior, in der Perspektive „der Lage von älteren Menschen und ihrer sozialen und lebenstechnischen Situation, spricht man immer öfter von der Notwendigkeit des Einsatzes des Hilfeleistungsmodells, der Unterstützung als ein Netzwerk existenzieller Verbindungen des Menschen mit der lokalen und familiären

\footnotetext{
5 Polnisches Sozialhilfegesetz vom 29. November 1990, Art. 8, s. 5.

6 http://www.ipsir.uw.edu.pl/UserFiles/File/Rekrutacja/Miedzynarodowa_definicja_pracy_socjalnej.pdf [Zugang am 12.03.2016].

7 J. Młyński, Człowiek wobec pomocy... Zarys pracy socjalnej, Tarnów 2009, s. 25.

8 D. DuBois, K. K. Miley, Praca socjalna. Zawód, który dodaje sił, Warszawa 1996, s. 141 .
} 
Umgebung. In der Analyse des Verbindungsnetzwerkes eines älteren Menschen mit seiner Soziosphäre, im breit gefassten Sinne des Wortes, ist eine Schlüsselkategorie die Leistung von Sozialhilfe und der mit ihm prozessual nahe verwandten Unterstützung im Bezug auf Einzelpersonen, Gruppen, Institutionen" ${ }^{\text {“9. }}$

Im Rahmen der Sozialarbeit sind ein paar Formen der Hilfe zu nennen, und zwar:

- Nothilfe (Rettungswesen),

- Vorsorge - eine Form, welche in Lebenssituationen geleistet wird, in welchen vom Unglück betroffene Menschen nicht imstande sind, die Schwierigkeiten zu überwinden, oder ausreichend Kräfte zu diesem Zweck haben;

- Hilfe - Aktivitäten, welche das Gedeihen sowohl von Personen, die aus irgendwelchem Grund in Gefahr stehen, als auch aller anderer Gesellschaftsmitglieder, unterstützen sollen, z. B. Familienberatungsstellen;

- Soziale Kompensation - ein Ausgleich der Mängel in der Umgebung, welche ein Gedeihen einer Einzelperson oder einer Gruppe beeinträchtigen, z. B. Ersatzfamilien ${ }^{10}$.

Ein Sozialarbeiter kann bei seiner Arbeit drei Methoden der Sozialarbeit einsetzen, und diese sind: die individuelle Methode, die Gruppenmethode sowie die Bildung einer Lokalgemeinschaft. Bei der Arbeit mit Senioren eignet sich individuelle Arbeit, welche eng an die Bedürfnisse des einzelnen angepasst ist, am besten, wenn möglich, bei dem Wohnsitz des Betroffenen. Diese Methode lässt beobachten, in welchen Grenzen eine gegebene Person aktiviert werden kann. Der Sozialmitarbeiter versucht daher, dem Senioren und der Familie „den Alterungsprozess zu erklären, die Einzelheiten der für ältere Menschen typischen Krankheiten zu beschreiben, zu Hause angebotene Leistungen zu erklären, die Methoden der Bewältigung schwieriger Situationen zu beschreiben, sowie die Möglichkeit geben, die gespürten Sorgen und Traurigkeiten mit anderen zu teilen" ${ }^{\text {"sl1 }}$.

Vor Beginn der Arbeit mit Senioren, soll er sich mit ihren Bedürfnissen vertraut machen; diese umfassen: Elend, Zugehörigkeit, Liebe, Freundschaft, Unterstützung, Verständigung. Das wichtigste Bedürfnis ist Gesundheit, unter den psychischen und physischen Aspekten. Ältere Menschen sind des Öfteren nicht im Stande, ihre grundlegenden Bedürfnisse aus der Grund einer schwierigen finanziellen Situation zu befriedigen. Sie leben auf Kosten des Verzichtens, was eine Nachteilsituation, und eine Sorge verursachende Situation ist. Daher werden in der Fachliteratur „Senioren in zwei Gruppen geteilt: die Gesunden (welche

9 A. Chabior, Wsparcie spoleczne i jego rodzaje, in: Starzenie się i starość w perspektywie pracy socjalnej, red. A. Chabior, A. Fabiś, J. K. Wawrzyniak, Warszawa 2014, s. 125.

10 http://www.dlastudentow.za.pl/formy_pracy_socjalnej.html [Zugang am 12.03.2016].

$11 \quad$ J.M. Młyński, Człowiek wobec pomocy..., op. cit., s. 168. 
größtenteils selbstständig Leben können und das System der informellen Sorge sowie formelle Sozialleistungen in Anspruch nehmen können) und die Kranken (die formale Hilfe benötigen) ${ }^{\star 12}$.

Die Leistungen für Menschen, welche bei Ihren Aufenthaltsorten verweilen, umfassen hauptsächlich Hilfe vor Ort, welche von einem Sozialarbeiter organisiert und eingesetzt wird. Die formelle Hilfe vor Ort kreist um: Finanzielle Hilfe, Sachleistungen, Dienstleistungen und institutionelle Hilfe. Finanzielle Hilfe wird in Rahmen von zweckgebundenen und periodischen Zuschüssen abgewickelt. Sachleistungen haben den Zweck, die grundlegenden Lebensbedürfnisse zu decken (Mittagessen, Lebensmittel, Kleidung, Schuhe, Brennmaterial). Dienstleistungen umfassen Aktivitäten der Sorge (Einkäufe, Hilfe bei mit der Hygiene verbundenen Tätigkeiten, Aufräumen des Hauses, Freizeitgestaltung). Institutionelle Hilfe umfasst Aufenthalte bei Sozialhilfeanstalten, Tageshilfestätten, usw. ${ }^{13}$.

Die Formen der zugunsten älterer Menschen geleisteter Sozialhilfe können auf zwei Ebenen zusammengefasst werden: Institutionelle Hilfe und Hilfe vor Ort. Diese Fragestellung wird von der folgenden Tabelle veranschaulicht.

Tabelle 1. Formen der Hilfe für ältere Menschen, welche auf zwei Ebenen abgewickelt wird

\begin{tabular}{|c|c|c|}
\hline$\Xi$ & $\begin{array}{l}\text { FINANZIELLE } \\
\text { HILFE }\end{array}$ & $\begin{array}{l}\text { Feste Zuschüsse (verbunden z. B. mit einer permanenten } \\
\text { Arbeitsunfähigkeit) } \\
\text { Periodische Zuschüsse } \\
\text { Zweckgebundene Zuschüsse (Medikamente, Brennmaterial, } \\
\text { Kleidung) } \\
\text { Sonderzuschüsse (Hilfe zur Selbstständigkeit aus Gründen } \\
\text { des Gefängnisaufenthaltes oder der Naturkatastrophen) }\end{array}$ \\
\hline 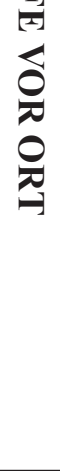 & $\begin{array}{l}\text { SACHLEISTUNGEN } \\
\text { UND } \\
\text { DIENSTLEISTUNGEN }\end{array}$ & $\begin{array}{l}\text { Sicherung von: Unterschlupf, Ernährung, Kleidung, } \\
\text { Begräbnis } \\
\text { Leistungen der sog. Sozialarbeit } \\
\text { Leistungen vor Ort: Sorge, Hygiene, Rehabilitation, Hilfe bei } \\
\text { Herstellung der möglichst breiten Selbständigkeit } \\
\text { Sicherung des notwendigen Transports, Organisation und } \\
\text { Hausführung. } \\
\text { Aktivitäten, welche diverse Unterstützungsformen in } \\
\text { sich verbinden, wie: Kriseninterventionen, individuelle } \\
\text { Obdachlosen-Hilfsprogramme, Sozialverträge. }\end{array}$ \\
\hline
\end{tabular}

12 D. DuBois, K.K. Miley, Praca socjalna ..., op. cit., s. 142-143.

13 Vgl. J. Kędzior, Współczesna wyzwania..., op. cit., s. 178. 


\begin{tabular}{|c|c|c|}
\hline & HALBOFFEN & $\begin{array}{l}\text { Hilfe und Unterstützung, welche von Tagesstätten wie } \\
\text { Tageshilfestätten, Hilfsstätten (Übernachtungsstätten, } \\
\text { Tagesstätten, lokale Selbsthilfeanstalten) geleistet werden. }\end{array}$ \\
\hline 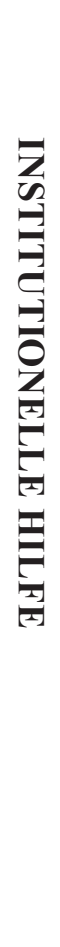 & GESCHLOSSEN & 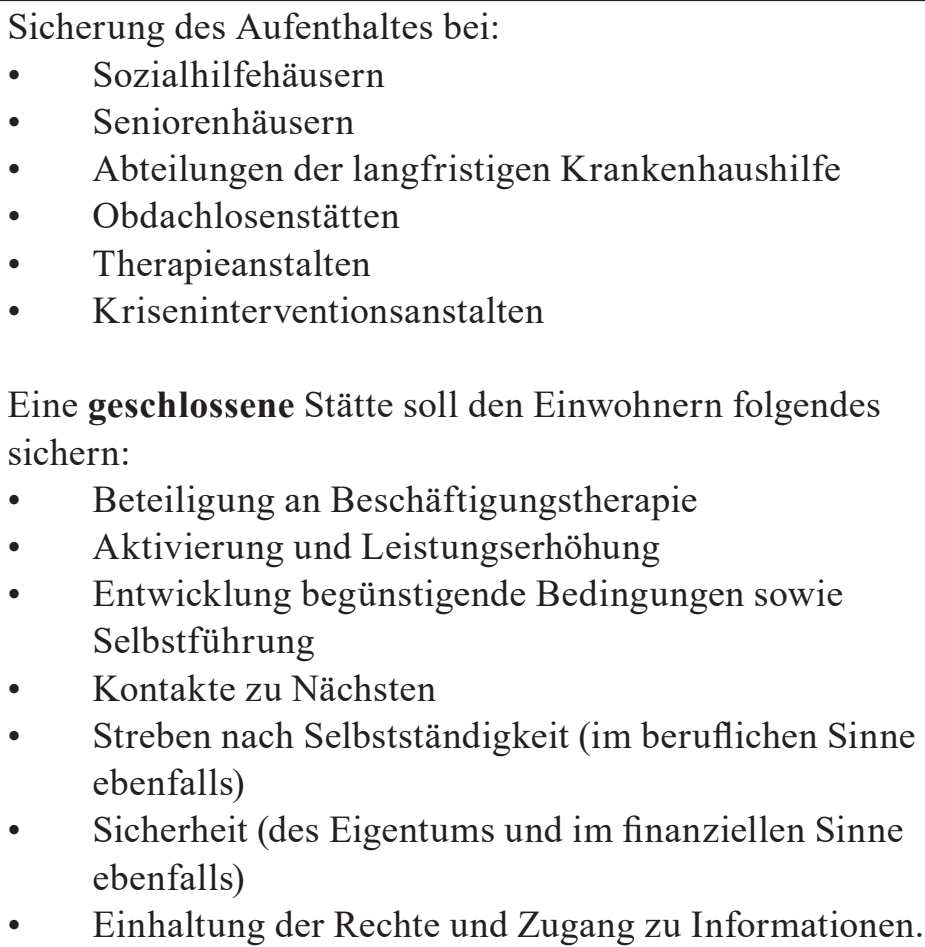 \\
\hline
\end{tabular}

Quelle: J. K. Wawrzyniak, Instytucje pomocowe i organizacje pozarzadowe w pracy na rzecz osób starszych [Hilfsorganisationen und Nichtregierungsorganisationen in der Arbeit zugunsten älterer Menschen] [in:] Starzenie się i starość w perspektywie pracy socjalnej, red. A. Chabior, A. Fabiś, J. K. Wawrzyniak, Warszawa 2014, s. 150.

Aus der obenstehenden Tabelle geht hervor, dass Sozialarbeit drei grundlegende Formen annimmt: diejenige vor Ort, von halboffenen und geschlossenen Stätten, auch institutionelle Hilfe genannt. Jede dieser Formen bleibt ein wichtiger Teil der Hilfe für ältere Menschen.

\section{Aufgaben eines Sozialarbeiters bei Anstalten für ältere Personen}

Ein Sozialarbeiter unternimmt die Arbeit bei vielen Institutionen und Organisationen sowie bei Lokalbehörden. Die wichtigsten Institutionen, welche Sozialhilfe für Senioren in Polen leisten, sind: Gemeinde-Sozialhilfeanstalten, Sozialhilfehäuser, Tagesstätten.

Ältere Menschen, welche bei einem Sozialhilfehaus verbleiben, können auf Unterstützung eines Sozialarbeiters hoffen. Es passiert oft, dass solche Menschen nur ungern in Sozialhilfehäusern bleiben, dass sie sich von der Familie verlassen, 
verworfen fühlen, es ist aber in der Wirklichkeit nicht der Fall, da die Familie in den meisten Fällen schon alle ihre Möglichkeiten der Fürsorge für einen Senior, seien sie finanzieller oder psychischer Natur, ausgeschöpft hat. Familien leiden auch aus der Ursache der Überweisung ihrer Nächsten in Sozialhilfehäuser, es ist jedoch in den meisten Fällen unabdingbar ${ }^{14}$.

Sozialarbeiter bei einem Sozialhilfehaus sollen Hilfe nicht nur den Bewohnern des Hauses leisten, aber ihrer Familien ebenso. Daher stützen sie ihre Aktivität auf festen Werten, zu denen gehören die Würde des Menschen, seine grundlegenden Bedürfnisse sowie Chancengleichheit. Darüber hinaus, eine wichtige Aufgabe eines Sozialarbeiters bei einem Sozialhilfehaus ist sie Hilfe für den Senior beim Verstehen des Übergangs zur Vorsorgestätte als einer weiteren Phase des menschlichen Lebens, sowie Begleitung über den ganzen Aufenthalt dieser Person bei der entsprechenden Stätte. Ferner leisten Sozialarbeiter Unterstützung bei der Adoption einer neuen Person, Unterhalt der Kontakte mit der Familie, Hilfe bei Alltagsangelegenheiten, Versorgung mit Kleidung, Schuhen, Ausstattung, sowie die Organisation von Treffen im Bereich der fachlichen rechtlichen und gesundheitlichen Beratung. Er befasst sich hauptsächlich mit der Abwicklung der Befugnisse der Bewohner, der Verbesserung der Lebensqualität, der Herstellung guter zwischenmenschlicher Beziehungen. er ist für die Aktivierung der Bewohner zuständig, und zwar durch diverse Formen der Therapie ${ }^{15}$.

Eine wichtige Aufgabe eines Sozialarbeiters ist es, eine Diagnose des jeweiligen betreuten Bewohners aufzustellen, und von daher „braucht der Sozialarbeiter eine tiefgreifende Analyse der Lage des Betreuten durchzuführen, das zu überwältigende Problem zu beschreiben sowie ein Hilfs- und Abwicklungsprogramm aufzustellen. Auf diese Weise, durch Einsatz seiner Kenntnisse, versucht er die Fähigkeit des Betreuten, seine Probleme selbstständig zu lösen, zu stärken ${ }^{\text {“16. }}$. In der Erfüllung seiner Funktion eines Erstkontaktarbeiters, Beteiligt er sich an die Sitzungen des therapeutischen und betreuerischen Teams ${ }^{17}$. Aus diesem Grund sind die Leistungen eines Sozialarbeiters bei einem Sozialhilfehaus grundlegend: Information über die Möglichkeit des Aufenthaltes eines Senioren bei einem Sozialhilfehaus und über verwandte Angelegenheiten, Hilfe bei der Adaptation eines Betreuten bei einer Hilfestätte, Hilfe bei Problemlösung, Aktivierung eines Betreuten, Anspornen zu

14 Vgl, R.A. Skidmore, M.G. Thackeray, Wprowadzenie do pracy socjalnej, Katowice 1998, s. 272.

15 Vgl. A. Mielczarek, Człowiek stary $w$ domu pomocy społecznej z perspektywy polityki społecznej i pracy socjalnej, Toruń 2010, s. 351.

16 Op. cit., s. 351.

17 Op. cit., s. 329-338. 
Selbstständigkeit, Hilfe bei Abwicklung verwaltungstechnischer Angelegenheiten, Zusammenarbeit mit Institutionen, Unterhaltung der Beziehungen zu der Familie, Freizeitgestaltung, Erklärung der fortschreitenden, mit dem Alter verbundenen Änderungen ${ }^{18}$.

In diesem Kontext soll er auch Respekt für die Würde eines Bewohners, dessen Selbstbestimmung und Verantwortung für das eigene Leben, die Einhaltung der Regeln der Vertraulichkeit, Objektivität sowie Anständigkeit bei der Information über seine Rechte im Auge behalten. Darüber hinaus soll seine Welt des Alterns als axiologisch richtig, mit dem Vergehensprozess verbunden ${ }^{19}$.

Einen weiteren Aufenthaltsort für ältere Menschen sind Tageshilfestätten, deren Aufgabe es ist, Betreuung und emotionale Fürsorge am Tag zu leisten. Diese Stätten leisten Pflege nur über einen bestimmten Zeitraum, erlauben es älteren Menschen, viele nicht nur grundlegende, aber auch hochrangige Bedürfnisse zu befriedigen. Ein Sozialarbeiter erfüllt dabei die Rolle eines Bindeglieds zwischen dem von den Institutionen angebotenen Programm auf der einen Seite, und der Familien auf der anderen Seite, sowie zwischen den einzelnen Stätten und Sozialhilfeagenturen. Diese Leistungen erleichtern es der Familie, die Fürsorge über den ganzen Tag zu leisten, weist institutionelle Hilfe zurück oder erlaubt es, sie vollständig zu vermeiden, gleichzeitig aber es erlaubend, unter den Senioren den höchsten Grad an physische und emotionale Aktivität zu behalten ${ }^{20}$. Darüber hinaus informiert er die Senioren und ihre Familien über den Aktivitäten der Stätte und hilft bei den verwaltungstechnischen Angelegenheiten vor Annahme eines Seniors. Die Aufgaben einer Tageshilfestätte stützen sich auf die Sicherung von einem Aufenthalt, Essen, Fürsorgeleistungen, Beschäftigungstherapie, Beteiligung an Interessenkreise, Freizeitgestaltung auf eine sichere und würdige Weise sowie Formen der Therapie durch Bewegung ${ }^{21}$.

Im Rahmen der geleisteten Unterstützung erfordert Hilfe für Personen das Öffnen eines Menschen auf den Anderen, das Interesse für sein Schicksal sowie die Herstellung einer gemeinsamen Kommunikationsebene. Der Betreuer-Freund ist „die gefragteste Methode der Hilfe, welche eine für Senioren Hilfe leistende Person einsetzen soll. Fachliche Hilfeleistung ist schwierig, man stößt auf viele Schwierigkeiten, wie: zwischenmenschliche Beziehungen, Engagement in das

18 J. Młyński, Praca socjalna wobec osób starszych na tle zmieniającej się rzeczywistości, in: Senior i rodzina, ed. M. Banach, A. Szwedzik, Kraków 2013, s. 30.

19 Vgl. M. Mielczarek, Człowiek stary w domu ..., s. 350.

20 R.A. Skidmore, M. G. Thackeray, Wprowadzenie..., s. 275-276.

21 Vgl. A. Leszczyńska-Racher, Człowiek starszy i jego wspomaganie - w strone pedagogiki starości, Olsztyn 2007, s. 163. 
Leben eines Anderen, die Fähigkeit des Lenkens der Aufmerksamkeit auf einen einzelnen Betreuten“222. In diesem Kontext ist das Bedürfnis an professionelle Hilfe mit den entsprechenden Kompetenzen von großer Bedeutung.

Nach A. Mielczarek ist ,professionelle Hilfeleistung ein fließender, sich verändernder Prozess. Man kann in der Tat nie sicher sein, dass alle Bestandteile der Arbeit mit einem Betreuten, Kunden oder Einwohner überarbeitet wurden. Gleichzeitig aber macht dies diese Arbeit besonders interessant, da es erfordert die Aktivierung geistiger und emotionaler Kräfte, und verleiht Zufriedenheit, wenn man bei dieser Menge an Bedingungen effektiv zu sein vermochte“ ${ }^{\text {“23. }}$.

Im Rahmen der von Sozialarbeitern unternommenen institutionellen Tätigkeiten soll in die Liste ihrer Aktivität in Bezug auf ältere Personen folgendes eingeführt werden:

- „eine Analyse und eine Beurteilung der Phänomene, welche zur Beantragung von Hilfe führen,

- Unterbreiten von Informationen und Hilfe im Bezug auf Problemlösung sowie Möglichkeiten der Verbesserung der eigenen (sozialen, finanziellen, rechtlichen) Situation,

- das Anspornen der sozialen Aktivität der Senioren und ihre Beteiligung an Selbsthilfemaßnahmen (z. B. in der nächsten Umgebung),

- die Initiation (neuer) Formen von Hilfe für Einzelpersonen und Gruppen unter schwierigen Lebensbedingungen,

- die Beteiligung an die Entwicklung und Umsetzung (lokaler) Programme der Hilfe und Unterstützung der sozialen Gruppen und Gemeinschaften,

- Hilfe bei Zugang zu Leistungen, zu Hilfe und zu Beratung, welche mit der besonderen Situation eines Einzelnen oder einer Gruppe verwandt sind,

- Zusammenarbeit mit Institutionen und Fachleuten aus anderen Gebieten, deren Engagement bei Problemlösung behilflich sein wird (darunter auch Devianzen und Elend),

- Hilfe bei Gewinnung von Mitteln und Fähigkeiten der Bewältigung schwieriger Situationen, Sensibilisierung auf die Bedürfnisse älterer Menschen,

- Vereinfachung des Kontaktes der Senioren mit anderen Einzelpersonen, Gruppen oder Institutionen“"24.

22 A. Mielczarek, Człowiek stary..., s. 327.

23 Op. cit., s. 370.

24 Vgl. J.K. Wawrzyniak, Instytucje pomocowe i organizacje pozarzadowe w pracy na rzecz osób starszych, in: Starzenie się i starość..., s. 150. 
Zweifellos erfüllt der Sozialarbeiter eine wichtige Rolle in dem Prozess der Unterstützung der Senioren. In der Tat, bei Unternahmen der Zusammenarbeit mit diversen Hilfsanstalten sowie Institutionen beteiligt er sich an dem Prozess der Hilfeleistung und Unterstützung der Senioren durch Teilnahme an die Zuweisung von finanzieller, dienstleistungstechnischer oder institutioneller Hilfe. Die erste genannte Art der Hilfe bezieht sich auf Unterstützung in der Form diverser Zuschüsse sowie Sachleistungen. Die zweite Art der Hilfe sind hauptsächlich Fürsorgeaufgaben, welche bei der Befriedigung tagtäglicher Lebensbedürfnisse notwendig sind, wie Einkäufe, Vorbereitung von Essen, Waschen, Aufräumen, Hilfe bei persönlicher Hygiene. Es umfasst wiederum die Institutionelle Hilfe die Unterbringung einer älteren Person bei einer entsprechenden Institution, bei einem Sozialhilfehaus oder einer Tagesstätte ${ }^{25}$.

\begin{abstract}
Abschluss
Zusammenfassend kann gesagt werden, dass die Leistung von Sozialarbeit eine enorm wichtige Unternehmung zugunsten Senioren und alternde Gesellschaften ist. $\mathrm{Zu}$ den grundlegenden Aufgaben der Sozialarbeiter im Bezug auf ältere Menschen gehören: Die Aufklärung der Senioren im Bereich des Alterungsprozesses und der dabei vorkommenden Änderungen, die Ausrichtung an entsprechende Institutionen, Hilfe bei dem Ausfüllen diverser Arten von Bewerbungen und offiziellen Schreiben, Motivierung der Senioren zu selbstständiger Problemlösung, die Veranstaltung diverser Hilfeformen, das Anspornen der gesellschaftlichen Aktivität, die Inspiration zu Selbsthilfe, Hilfe bei Befriedigung der Lebensbedürfnisse.

Obwohl einer der ersten Orte der Unterstützung für Senioren die Familie als die eigene Referenzgruppe fungiert, brauchen viele solcher Menschen institutionelle Unterstützung. Viele Familien verwerfen zwar nicht ihre eigenen Älteren, aber aus Ursache ihrer Krankheit und der Notwendigkeit der Hospizhilfe und Palliativversorgung, sind sie dazu gezwungen, eine solche Person am Lebensende an eine Gesundheitsinstitution zu übergeben ${ }^{26}$. Ein Sozialarbeiter ist das Bindeglied zwischen Senioren und Institutionen. Er leistet Informationen über Programme, an welchen Senioren und ihre Familie sich beteiligen können. Darüber hinaus baut er in der lokalen Umgebung die soziale Sicherheit dieser Gruppe durch Verhinderung der Marginalisierung der Senioren aus, er hilft sie zur Selbstentwicklung durch

25 Vgl. J. Brągiel, P. Sikora, Praca socjalna wobec rzeczywistych i potencjalnych zagrożeń człowieka, Opole 2005, s. 78.

26 Vgl. A. Fabis, Opieka nad osobami starszymi, in: Starzenie się i starość..., s. 103. Vgl. A. Fabis, Opieka paliatywna, in: Starzenie się i starość..., s. 113.
\end{abstract}


Vereinfachung des Zugangs zu diversen Formen des permanenten Lernens, zu Erholung und zu Kultur- und Bildungsanstalten. In diesem Kontext ist die Sozialarbeit eine professionelle Perspektive der Unterstützung funktionsgestörter Familien, Familien mit mehreren Problemen, und vor allem, älterer Menschen ${ }^{27}$.

Es soll daher viel Mühe gegeben werden, damit ältere Menschen in der heutigen Gesellschaft ein besseres Leben führen könnten. Diese können aus der bloßen Ursache, dass sie alt sind, nicht daraus ausgeschlossen werden. Eine Person im fortgeschrittenen Alter braucht einen Freund, Begleiter, sorgfältigen Zuhörer, mit welchem sie ihre Zweifel besprechen könnte, und durch welchen sie andere, bessere Dimensionen des Alters sehen könnte. Wir alle werden eines Tages alt, und wir alle werden mit Würde leben und weiterhin von der Gesellschaft akzeptiert werden wollen, da sollen wir unsere Einstellung gegenüber ältere Personen überdenken, sodass wir diese nicht zufällig verletzen, verwerfen, diskriminieren. Es scheint, dass diese Arbeit diese Möglichkeiten den Senioren vor Augen gestellt hat, und dass sie es erklären könnte, dass der Alterungsprozess sehr lang ist, und dass darüber hinaus jede einzelne Person, als Gesellschaftsmitglied, nach ihren eigenen Möglichkeiten den Senioren helfen soll, sodass dieser Prozess für sie nicht nur schön, aber auch axiologisch wundervoll sein wird.

\section{Bibliographie}

Błędowski P., Lokalna polityka społeczna wobec ludzi starszych [Lokale Sozialpolitik gegenüber ältere Menschen], Warszawa 2002.

Brągiel J., Sikora P., Praca socjalna wobec rzeczywistych i potencjalnych zagrożeń człowieka [Sozialarbeit im Lichte echter und potenzieller Gefahren für den Menschen], Opole 2005.

Chabior A., Wsparcie społeczne i jego rodzaje [Sozialunterstützung und ihre Formen], in: Starzenie się $i$ starość w perspektywie pracy socjalnej, Red. A. Chabior, A. Fabiś, J. K. Wawrzyniak, Warszawa 2014, s. 125-132.

DuBois D., Miley K., Praca socjalna. Zawód, który dodaje sit [Sozialarbeit. Ein kraftverleihender Beruf], Warszawa 1996.

Fabis A., Opieka nad osobami starszymi [Sorge für ältere Menschen], in: Starzenie się $i$ starość $w$ perspektywie pracy socjalnej, red. A. Chabior, A. Fabiś, J.K. Wawrzyniak, Warszawa 2014, 103-112.

27 Vgl. J. Szmagalski, Profesje we współczesnej perspektywie teoretycznej a wyzwania profesjonalizacji pracy socjalnej w Polsce, in: Profesjonalna praca socjalna. Nowy paradygmat czy niedokończone zadanie, ed. K. Piątek, K. Szymańska- Zybertowicz, Toruń 2011, s. 17. 
Kędzior J., Współczesne wyzwania pracy socjalnej [Gegenwärtige Herausforderungen der Sozialarbeit], Toruń 2006.

Leszczyńska-Racher A., Człowiek starszy i jego wspomaganie - w strone pedagogiki starości [Ein älterer Mensch und seine Unterstützung - zur Pädagogik des hohen Alters], Olsztyn 2007.

Mielczarek A., Człowiek stary w domu pomocy spolecznej z perspektywy polityki społecznej i pracy socjalnej [Ein alter Mensch in einem Sozialhilfehaus aus Sicht der Sozialpolitik und Sozialarbeit], Toruń 2010.

Młyński J., Człowiek wobec pomocy... Zarys pracy socjalnej [Der Mensch und Hilfe... Ein Abriss der Sozialarbeit], Tarnów 2009.

Młyński J., Praca socjalna wobec osób starszych na tle zmieniajacej się rzeczywistości [Sozialarbeit im Bezug auf ältere Personen vor dem Hintergrund einer sich verändernden Wirklichkeit], in: Senior i rodzina, red. M. Banach, A. Szwedzik, Kraków 2013.

Polnisches Sozialhilfegesetz vom 29. November 1990, Art. 8.

Rosset E., Ludzie starzy. Studium demograficzne [Alte Menschen. Ein demografisches Studium], Warszawa 1967.

Skidmore R.A., Thackeray M.G., Wprowadzenie do pracy socjalnej [Einführung in die Sozialarbeit], Wydawnictwo Śląsk, Katowice 1998.

Szatur-Jaworska B., Błędowski, P., Dzięgielewska M., Podstawy gerontologii społecznej [Grundlagen der Sozialgerontologie], Warszawa 2006.

Szmagalski J., Profesje we współczesnej perspektywie teoretycznej a wyzwania profesjonalizacji pracy socjalnej $w$ Polsce [Berufe in der heutigen theoretischen Perspektive und die Herausforderungen der professionellen Sozialarbeit in Polen], in: Profesjonalna praca socjalna. Nowy paradygmat czy niedokończone zadanie, red. K. Piątek, K. Szymańska-Zybertowicz, Toruń 2011, s. 17-36.

Wawrzyniak J. K., Instytucje pomocowe i organizacje pozarzadowe w pracy na rzecz osób starszych [Hilfsorganisationen und Nichtregierungsorganisationen in der Arbeit zugunsten älterer Menschen], in: Starzenie się i starość w perspektywie pracy socjalnej, red. A. Chabior, A. Fabiś, J.K. Wawrzyniak, Warszawa 2014, s. 133-150.

Zych A., Stownik gerontologii społecznej [Wörterbuch der Sozialgerontologie], Warszawa 2001.

http://www.dlastudentow.za.pl/formy_pracy_socjalnej.html[Zugang am 12.03.2016].

http://www.ipsir.uw.edu.pl/UserFiles/File/Rekrutacja/Miedzynarodowa_definicja_pracy_socjalnej.pdf [Zugang am 12.03.2016]. 


\title{
Józef Młyński: Support for the elderly as part of social work in Poland
}

\begin{abstract}
Summary
Contemporary society is very diverse. Increasingly, there is a lack of interpersonal understanding and the man lives in constant changes and haste. It must be admitted that the society of rapid development and change is difficult to live in, especially for the elderly, who often feel rejected and useless while entering the so-called aging period.

Forms of helping people, also in the period of aging, include social work. That is why this article is situated in the area of senior support and concerns the clarification of basic issues concerning forms of assistance to the elderly and the role of the social worker in supporting seniors. The aim is to show the value of social work benefits offered to elderly people (seniors) as instruments of help and support to this group of people.
\end{abstract}

Keywords: old age, seniors, social work, support, help, benefits 